\title{
A Rare Case of Primary Gastric Melanoma
}

\author{
Maciej Wiewiora $^{1} \cdot$ Katrzyna Steplewska ${ }^{2} \cdot$ Jerzy Z. Piecuch $^{3} \cdot$ Jerzy Piecuch $^{1}$
}

Received: 22 February 2019 / Accepted: 11 September 2019/Published online: 25 October 2019

(C) The Author(s) 2019

\begin{abstract}
Primary melanomas in the gastrointestinal tract are extremely rare, with an estimated prevalence of 0.5 to 1 case per million. Primary mucosal melanomas of gastric origin account for approximately 1-3\% of all primary melanomas in the gastrointestinal tract. We present the case of a patient who underwent surgery due to primary gastric melanoma.
\end{abstract}

Keywords Malignant melanoma $\cdot$ Primary gastric melanoma $\cdot$ Surgery

\section{Introduction}

Malignant melanoma is a cancer originating from pigment cells and is most often located in the skin. Melanoma malignum is rarely found in the alimentary tract, and malignant gastrointestinal melanomas are usually metastatic [1]. Primary gastrointestinal melanomas (PGIMs) occur at a frequency of 0.5 to 1 case per million, depending on race and latitude. Primary gastric melanomas (PGM) account for $12.7 \%$ of all PGIMs $[2,3]$.

We present the case of a patient who underwent surgery due to PGM.

\section{The Case Report}

A 66-year-old woman was diagnosed with anaemia and weight loss. The patient's history included chronic gastritis, kidney stones and coronary heart disease. She had previously undergone implantation of a cardiac pacemaker, implantation of a biological valve in the aorta and excision of her gallbladder. The endoscopic examination revealed a stomach tumour

Maciej Wiewiora

m-wiewiora@tlen.pl

1 Department of General and Bariatric Surgery and Emergency Medicine in Zabrze, Medical University of Silesia, ul. Sklodowskiej-Curie10, 41-800 Zabrze, Poland

2 Department of Pathology, Institute of Medicine, University of Opole, Opole, Poland

3 Student Scientific Society, Department of General and Bariatric Surgery and Emergency Medicine in Zabrze, Medical University of Silesia, Katowice, Poland that was approximately $5 \mathrm{~cm}$ in diameter. Histopathological examination of the tumour samples suggested the diagnosis of malignant melanoma [IHC: CK (-), CHR (-), SYN (-), HER2 (-), VIM (+), HMB45 (+)]. Due to an initially insufficient amount of tissue, the endoscopic examination was repeated, and more samples were taken. The samples revealed that the tumour was composed of epithelioid cells that lacked cohesive features, had abundant cytoplasm, and had nuclei containing vacuoles and large nucleoli. The tumour cells were positive for Melan A expression and negative for CK AE1/ AE3 expression (Fig. 1a, b). Thus, the diagnosis of malignant melanoma was confirmed. The patient had dermatological and ophthalmological consultations as well as imaging examinations that excluded the presence of melanoma outside the gastrointestinal tract. Computed tomography (CT) of the abdominal cavity showed the stomach corpus tumour without infiltration of adjacent organs or enlarged lymph nodes (Fig. 2a). There were also no other nodular changes found in the remaining part of the digestive tract. CT of the chest excluded melanoma metastases to the lungs. The patient underwent surgery, resection of the stomach, spleen and greater omentum and D2 lymphadenectomy with additional excision of the lymph nodes of the liver cavity were performed (Fig. 2b, c). The oesophagus was anastomosed with the jejunum end to end with mechanical sutures (stapler EEA 25) on the Roux-en-Y loop. The postoperative histopathological examination revealed a white, solid, ulcerated tumour $(5 \times 5 \times 1.7 \mathrm{~cm})$ on the anterior wall of the stomach. The tumour had infiltrated the mucous membrane and the surface layer of the muscle membrane. The neoplastic infiltration consisted of epithelioid cell clusters with abundant cytoplasm, intranuclear vacuoles and prominent nuclei and a 12M/10HPF mitotic index. The tumour cells showed Melan A expression and no cytokeratin 
Fig. 1 Histopathological examination of the tumour samples before surgery. a Tumour cells showing Melan A expression (magnification $\times 200$ ). b Tumour cells without CK AE1/ AE3 expression (magnification $\times$ 400)
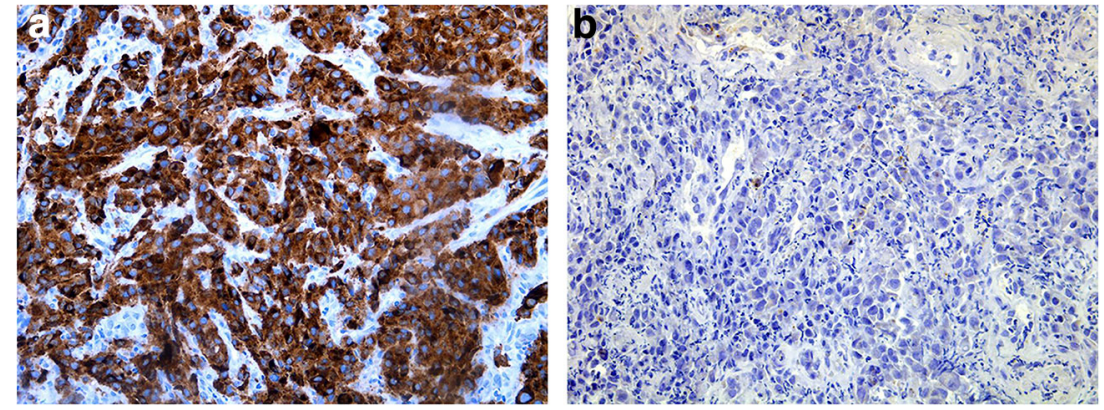
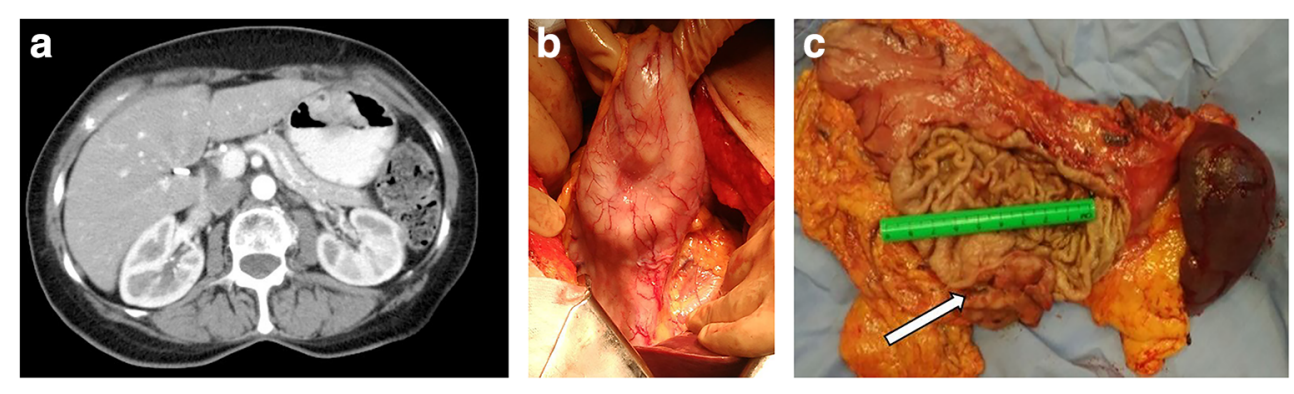

Fig. $2 \mathrm{CT}$ of the abdominal cavity and intraoperative view. The tumour of the anterior stomach wall. a Cross-sectionCT. b Tumour modelling the stomach wall. c Excised stomach with a tumour (arrow), the spleen and the greater omentum
(AE1/AE3) expression. Based on the histopathology and immunohistochemistry, malignant melanoma was diagnosed. No features suggesting angioinvasion or neuroinvasion were found. A melanoma metastasis was found in one lymph node of the greater curvature. The surgical margins, greater omentum and spleen were free from melanoma infiltration. The postoperative course had no complications. After the oncological consultation, it was determined that she qualified for chemotherapy. Due to the patient's lack of consent, further treatment was not pursued. Currently, 18 months after the surgery, no recurrence of the disease has been observed.

\section{Discussion}

PGIM is a very rare gastrointestinal cancer with unidentified aetiology. There are two theories regarding the formation of primary melanoma lesions in the alimentary tract. The first points to the possibility of the transformation of nerve cells from the APUD family (amine precursor uptake and decarboxylation) into melanocytes, which then undergo neoplastic transformation [4]. The second theory assumes the possibility of melanocyte precursor migration into the digestive tract. This hypothesis is based on observations of benign melanosis, which may be accompanied by the development of gastrointestinal tumours [5]. The diagnosis of PGM can be difficult because the clinical symptoms are nonspecific, and it is difficult to distinguish from other cancers and metastatic melanomas, which account for the vast majority of melanomas detected in the gastrointestinal tract, of which approximately $20 \%$ are metastases in the stomach [3]. The clinical criteria for the diagnosis of PGIM include the absence of simultaneous or previously removed melanomas or atypical melanocytic skin lesions and melanoma metastases outside the gastrointestinal tract [6]. Due to the invasive nature of the disease, the survival of patients with PGIM is still unsatisfactory and is usually shorter than 1 year. The average survival time from the diagnosis of the disease for patients with gastrointestinal melanoma is approximately 17 months, and for those with PGM, it is only 5 months. The basic method of treatment is surgery. It has been demonstrated that the survival times for patients who undergo surgery compared to those who do not are 12 months and 2 months, respectively [3]. The use of adjuvant chemotherapy may improve the results of treatment [7], whereas radiotherapy may be considered a method of palliative treatment [8].

\section{Compliance with Ethical Standards}

Conflict of Interest The authors declare that they have no conflict of interest.

Open Access This article is distributed under the terms of the Creative Commons Attribution 4.0 International License (http:// creativecommons.org/licenses/by/4.0/), which permits unrestricted use, distribution, and reproduction in any medium, provided you give appropriate credit to the original author(s) and the source, provide a link to the Creative Commons license, and indicate if changes were made. 


\section{References}

1. Manouras A, Genetzakis M, Lagoudianakis E, Markogiannakis H, Papadima A, Kafiri G, Filis K, Kekis PB, Katergiannakis V (2007) Malignant gastrointestinal melanomas of unknown origin: should it be considered primary? World J Gastroenterol 13:4027-4029

2. Bishop KD, Olszewski AJ (2014) Epidemiology and survival outcomes of ocular and mucosal melanomas: a population-based analysis. Int J Cancer 134:2961-2971

3. Cheung MC, Perez EA, Molina MA, Jin X, Gutierrez JC, Franceschi D, Livingstone AS, Koniaris LG (2008) Defining the role of surgery for primary gastrointestinal tract melanoma. J Gastrointest Surg 12: 731-738

4. Tabaie HA, Citta RJ, Gallo L, Biondi RJ, Meoli FG, Silverman D (1984) Primary malignant melanoma of the small intestine: report of a case and discussion of the APUD cell concept. J Am Osteopath Assoc 83:374-377
5. Horowitz M, Nobrega MM (1998) Primary anal melanoma associated with melanosis of the upper gastrointestinal tract. Endoscopy 30:662-665

6. Blecker D, Abraham S, Furth EE et al (1999) Melanoma in the gastrointestinal tract. Am J Gastroenterol 94:342733

7. Kim NR, Lee WK, Chung DH (2013) Primary gastric melanoma with rhabdoid features: a case report. Korean J Pathol 47:606-609

8. Slater JM, Ling TC, Slater JD, Yang GY (2014) Palliative radiation therapy for primary gastric melanoma. J Gastrointest Oncol 5:E22E26

Publisher's Note Springer Nature remains neutral with regard to jurisdictional claims in published maps and institutional affiliations. 